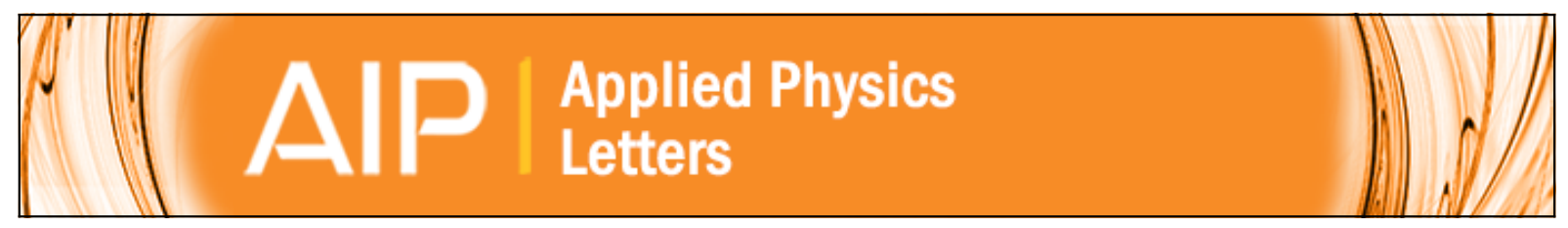

Bulk-grain resistivity and positive temperature coefficient of $\mathrm{ZnO}$-based varistors

D. Fernández-Hevia, J. de Frutos, A. C. Caballero, and J. F. Fernández

Citation: Applied Physics Letters 82, 212 (2003); doi: 10.1063/1.1534620

View online: http://dx.doi.org/10.1063/1.1534620

View Table of Contents: http://scitation.aip.org/content/aip/journal/apl/82/2?ver=pdfcov

Published by the AIP Publishing

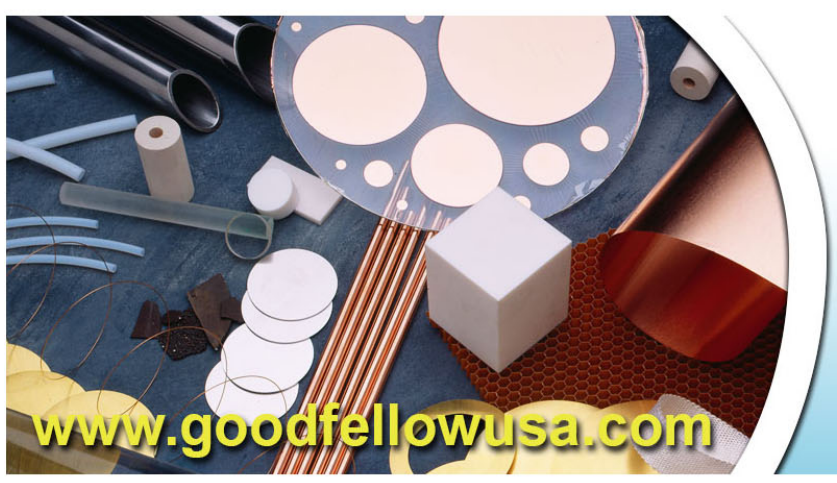

BODIFElIOU metals • ceramics • polymers composites $\cdot$ compounds $\cdot$ glasses Save $5 \% \cdot$ Buy online 70,000 products $\cdot$ Fast shipping 


\title{
Bulk-grain resistivity and positive temperature coefficient of ZnO-based varistors
}

\author{
D. Fernández-Hevia ${ }^{a}$ and J. de Frutos \\ E.T.S.I. Telecomunicación, Univ. Politècnica de Madrid, Ciudad Universitaria s/n, 28040 Madrid, Spain
}

\author{
A. C. Caballero and J. F. Fernández \\ Departamento de Electrocerámica, Instituto de Cerámica y Vidrio, CSIC, Campus de Cantoblanco, 28049 \\ Madrid, Spain
}

(Received 26 June 2002; accepted 11 November 2002)

\begin{abstract}
We analyze the conditions that allow the bulk-grain regions of a polycrystalline semiconductor to be explored through electrical measurements. The temperature dependence of grain resistivity in $\mathrm{ZnO}$ varistors $(300-430 \mathrm{~K})$ is presented, and a positive temperature coefficient is found. This is consistent with a free-carrier density approaching exhaustion, and an electron mobility controlled mainly by lattice (both optical and acoustical) scattering. No grain conductivity activation energy is to be found above room temperature and, therefore, ac-impedance techniques can be inadequate for the evaluation of grain conductivity and shallow donor activation energy. (C) 2003 American Institute of Physics. [DOI: 10.1063/1.1534620]
\end{abstract}

Polycrystalline semiconductors are interesting from the fundamental and technological points of view. ${ }^{1}$ Their nonlinear charge transport properties are described through a double Schottky barrier (DSB) model, ${ }^{2,3}$ interrelating three different microscopic regions within each grain (see Fig. 1): the grain boundary $(\mathrm{GB})$, the depletion region, and the bulkgrain region. A correct understanding of bulk-grain-related phenomena in polycrystalline semiconductors is important for at least the following reasons: (1) In overvoltage protection devices, grain resistivity is closely related to the highfield performance, ${ }^{4-6}$ which is of prime functional importance. (2) Applications as microwave materials ${ }^{1,7}$ require detailed knowledge of bulk-grain properties, as they control the high frequency response. (3) Grain resistivity is very likely to be affected by any species diffused into the bulk, hence providing clues about the shallow donor chemical nature, which in the $\mathrm{ZnO}$ case is an open and technologically relevant problem. ${ }^{8,9}$ (4) Grain resistivity correlates with freecarrier density, which is a necessary input for characterization techniques such as deep level transient spectroscopy. ${ }^{10}$

Despite this relevance, bulk-grain properties have not been extensively studied. For $\mathrm{ZnO}$ varistors, a few works have been published in which suitable electrical measurements directly yield grain resistivity; ${ }^{4,5}$ alternatively, an infrared reflectance technique ${ }^{6}$ was proposed that indirectly measures free-electron density and then converts it into grain resistivity. At zero applied field, the conditions for electrical measurements to yield meaningful information about grain interiors can be understood through the equivalent circuit of Fig. 2. Close to the dc limit, charge transport is dominated by thermionically emitted over-barrier currents. ${ }^{1-3}$ The dc resistance $\left(R_{\mathrm{dc}}\right.$ in Fig. 2$)$ has a negative temperature coefficient (NTC) due to the temperature dependence of the thermionic current: an Arrhenius plot of $R_{\mathrm{dc}}$ yields a thermal activation energy that can be correlated ${ }^{1,2,11}$ with the barrier height.

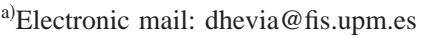

This dc transport mechanism is separated from the true GB dielectric response, which can be represented ${ }^{2}$ by a Debyelike $R_{\mathrm{GB}}-C_{\mathrm{GB}}$ series combination with a long, fielddependent relaxation time. ${ }^{1-3}$ Upon increasing frequency, the dielectric relaxations ${ }^{12,13}$ due to deep levels in the depletion layer (assumed to be Debye-like within the DSB model ${ }^{1-3}$ and represented by $R_{\mathrm{DL} 1,2}-C_{\mathrm{DL} 1,2}$ in Fig. 2), offer an easier path to charge transport, hence taking control of the current flow. Finally, as the capacitance $\left[C_{p}=\operatorname{Im}\left(Y^{*}\right) / \omega\right]$ of the DSB structure converges to its high-frequency (geometrical) value, ${ }^{2,3,14}$ the control of the transport properties moves from the depletion region to the bulk-grain region, and the bulkgrain resistance ( $R_{G}$ in Fig. 2 ) emerges, turning the equivalent circuit into a series $R_{G}-C_{\mathrm{HF}^{-}}-L$ combination. The geometrical capacitance $C_{\mathrm{HF}}$ appears at about $10^{8}-10^{9} \mathrm{~Hz}$, depending on the position of the highest-frequency loss peak. $^{14}$

Up to this point, the discussion is generally valid for any polycrystalline semiconductor. We now focus on $\mathrm{ZnO}$-based varistors. Electrical measurements up to $1 \mathrm{GHz}$ were performed on rod-shaped samples $\varnothing 1.5$ and $4 \mathrm{~mm}$ long, machined to this size in order to delay the onset of the geometri-

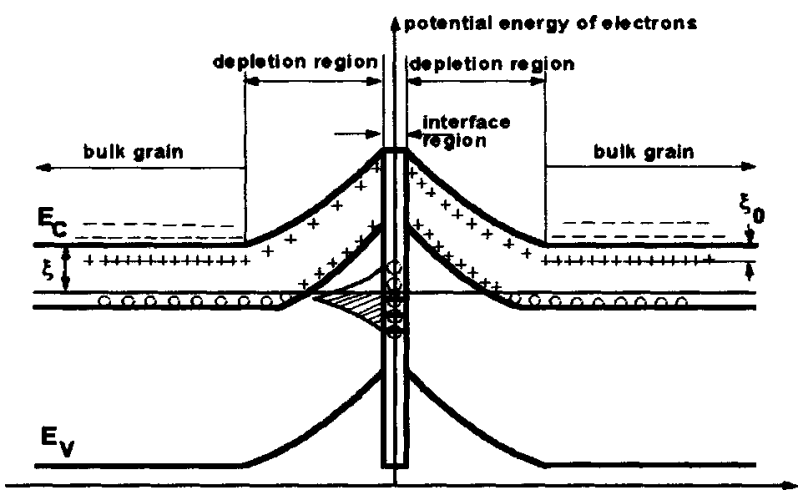

FIG. 1. Schematic energy band diagram of a double Schottky barrier. Open circles represent neutral states. $E_{V}$ is the valence band and $E_{C}$ is conduction band. The $x$-direction is orthogonal to the grain-boundary plane. 


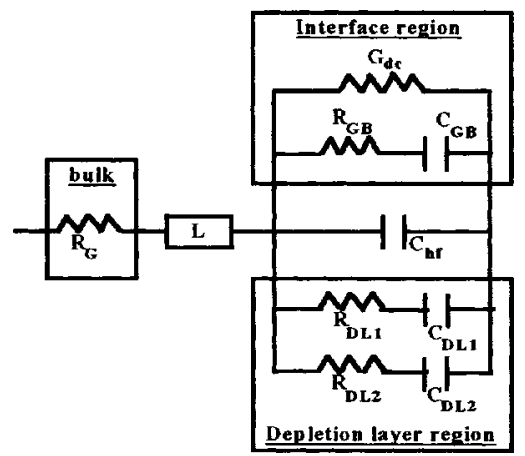

FIG. 2. Equivalent circuit for a double Schottky barrier structure over a very wide frequency range. $L$ is a built-in inductance due to the sample geometry and leads.

cal resonance, ${ }^{5}$ and to ensure complete penetration of the electromagnetic measuring signal. ${ }^{15,16}$ Samples were taken from commercial-grade, high-voltage (3 kV-rated, 3.33 $\mathrm{kV} / \mathrm{cm}$ residual voltage at $0.8 \mathrm{kA} / \mathrm{cm}^{2}$ ) $\mathrm{ZnO}$ varistors. Figure 3 shows the convergence with frequency of the series resistivity, measured at two different temperatures; $R_{G}$ values have been converted to $\Omega \mathrm{cm}$ by using microstructural information about the percentage of secondary phases in a polished cross section of the varistor material (approximately $22 \%$ in volume). The experimental points of Fig. 4 constitute our main experimental result: we find a small but welldefined positive temperature coefficient (PTC) behavior for the bulk-grain resistivity that has not been previously reported. To explain this result, we begin by writing the grain resistivity $\rho_{G}$ as

$$
\rho_{G}=1 /(n \mu e),
$$

where $n$ and $\mu$ are the free-carrier density and mobility in the grains, respectively. Before proceeding further, we need to clarify to what extent can we compare our high-frequency $\rho_{G}$ values with those calculated from the intrinsically dc Eq. (1). To this end, we use a simplified treatment of charge carrier inertia $^{17}$ that has been successfully applied to the high-frequency analysis of GaAs Schottky diodes. ${ }^{18,19}$ Within this model, free-carrier conduction in a semiconductor is represented by a complex conductivity

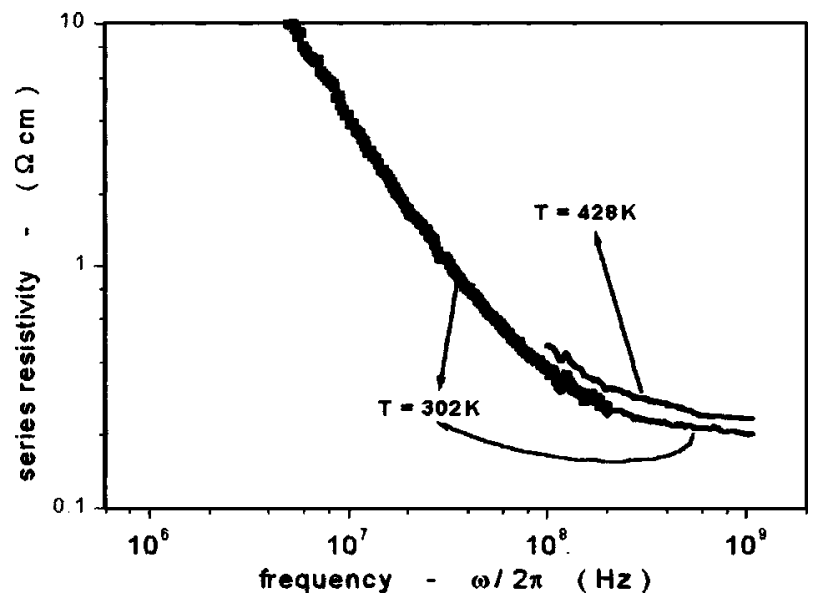

FIG. 3. Series resistivity for a typical $\mathrm{ZnO}$-based varistor. The lower trace has been obtained at $300 \mathrm{~K}$ over a wide frequency range, overlapping different measurements The upper trace has been obtained at $430 \mathrm{~K}$ in the high-frequency region.

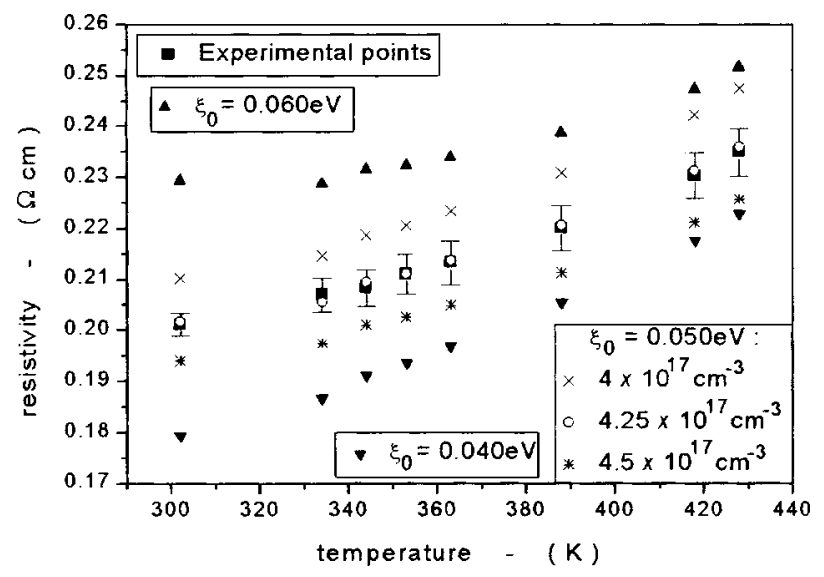

FIG. 4. Bulk PTC behavior in a typical $\mathrm{ZnO}$-based varistor. The figure shows the experimental points, along with calculated curves corresponding to $\xi_{0}=0.05 \mathrm{eV}$ and three different values of $N_{0}$ (the total shallow donor density). Two additional curves correspond to fixed $N_{0}=4.25 \times 10^{17} \mathrm{~cm}^{-3}$ and $\xi_{0}=0.04 / 0.06 \mathrm{eV}$.

$$
\sigma=\sigma_{0} /(1+i \omega \tau)
$$

where $\sigma_{0}=n \mu e$ is the dc conductivity, $\tau=\mu m^{*} / e$ is the average free carrier relaxation time, ${ }^{17}$ and $m^{*}$ is the free carrier effective mass. Hence, carrier inertia effects are negligible when $\omega \tau \ll 1$; introducing appropriate values for $\mathrm{ZnO}$ at room temperature ${ }^{20-22} \quad\left(m^{*}=0.27 m_{0}\right.$ and $\mu$ $\left.=140 \mathrm{~cm}^{2} / \mathrm{V} \mathrm{s}\right)$, this condition implies $\omega \ll 4 \times 10^{13} \mathrm{~Hz}$. Therefore, the electrical conductivity of bulk $\mathrm{ZnO}$ remains very close to its dc value up to the terahertz range, and we can safely compare our experimental results with the $\rho_{G}$ values to be calculated through Eq. (1). To do so, we note that reported $^{20-22} \mathrm{ZnO}$ mobility-temperature curves for a wide range of doping conditions, converge above room temperature due to the dominance of lattice over impurity scattering. Hence, we can select a reported $\mu-T$ curve $^{22}$ and, for each temperature, directly introduce the experimental mobility values in Eq. (1). Now, as thermodynamic calculations have shown that room temperature charge balance within varistor $\mathrm{ZnO}$ grains is maintained between electrons in the conduction band and ionized shallow donors, ${ }^{23}$ we can write the charge balance equation as

$$
n=N_{C} e^{-\xi / k_{B} T}=\frac{N_{0}}{1+2 \exp \left[\left(\xi_{0}-\xi\right) / k_{B} T\right]},
$$

where $N_{C}$ is the conduction band effective density of states, $N_{0}$ and $\xi_{0}$ are the shallow donor density and ionization energy, respectively, and $\xi$ is the Fermi level (see Fig. 1). Despite a long-standing controversy about the chemical nature of the shallow donor in $\mathrm{ZnO},{ }^{1,9,10,20-23}$ there seems to be general agreement upon its energy position, located about $0.05 \mathrm{eV}$ below the conduction band. ${ }^{20-23}$ Hence, assuming $\xi_{0} \approx 0.05 \mathrm{eV}$ in Eq. (3), we can solve for $\xi$ and $n$ at each temperature with the total shallow donor density $N_{0}$ as the only free parameter, to be adjusted in order for the quantity $1 /(n \mu e)$ to fit the experimental $\rho_{G}$ points. Figure 4 shows, along with the experimental points, those obtained through Eqs. (1) and (3) for three different values of $N_{0}$. The agree- 
ment obtained for $N_{0}=4.25 \times 10^{17} \mathrm{~cm}^{-3}$ is remarkable. The corresponding free-carrier density is $n=2.3 \times 10^{17} \mathrm{~cm}^{-3}$ at room temperature, in agreement with values obtained from reflectance measurements. ${ }^{6}$ We then conclude that, at room temperature and above, a free-carrier density approaching saturation and a lattice-scattering controlled mobility, render a PTC for the bulk-grain resistivity.

This PTC feature could explain the results obtained by Modine et al. ${ }^{24}$ who found that current amplitude decreased with successive current pulse applications; here, the behavior of the sample is bulk-grain dominated due to the barriersuppressing applied field, and Joule heating of the samples could manifest itself in an increase of grain resistance, leading to currents that decrease for constant applied voltage. ${ }^{24}$ This type of behavior is important for varistor stability under typical pulse and multipulse current testing, as prescribed by international standards.

As a by-product of the preceding analysis, we note that intermediate frequency (below $10 \mathrm{MHz}$ ) complex impedance spectroscopy and high-frequency intercept extrapolation, frequently used to obtain grain conductivity and shallow donor activation energy, ${ }^{12,25,26}$ can be inadequate for such purposes. This is so, because the DSB electrical response in this range is driven by deep level relaxations, ${ }^{12,13}$ and is not represented by a simple $R-C$ parallel combination, in series with $R_{G}$. Indeed, the data presented in Ref. 25 indicate that this procedure does not actually yield an approximation for the grain resistance, and the bulk conduction activation energies that have been obtained from this technique ${ }^{25,26}$ are in contradiction with the observed PTC behavior.

In conclusion, we have investigated the conditions that allow grain interiors in polycrystalline semiconductors to be explored with electrical measurements. Also, we have shown that $\mathrm{ZnO}$-based varistor materials exhibit a transition from NTC to PTC behavior, as the control of the electrical properties changes from the interface/depletion-layer regions to the bulk grain region. The NTC/PTC crossover can be de- fined either by a high enough frequency such that the material reaches its geometrical capacitance, or by an applied field that suppresses the grain boundary electrostatic barriers.

We acknowledge support from CICYT, project MAT2001-1682-C02-01/02.

${ }^{1}$ G. Blatter and F. Greuter, Semicond. Sci. Technol. 5, 111 (1990).

${ }^{2}$ G. E. Pike, Phys. Rev. B 30, 795 (1984).

${ }^{3}$ G. Blatter and F. Greuter, Phys. Rev. B 33, 3952 (1986).

${ }^{4}$ W. G. Carlson and T. K. Gupta, J. Appl. Phys. 53, 5746 (1982).

${ }^{5}$ L. M. Levinson and H. R. Phillipp, J. Appl. Phys. 47, 3116 (1976).

${ }^{6} \mathrm{H}$. R. Phillipp and L. M. Levinson, J. Appl. Phys. 47, 1112 (1976).

${ }^{7}$ N. McN. Alford, S. J. Penn, A. Templeton, X. Wang, and S. Webb, Industrial Ceramics 21, 21 (2001).

${ }^{8}$ A. F. Kohan, G. Ceder, D. Morgan, and C. G. Van de Walle, Phys. Rev. B 61, 15019 (2000)

${ }^{9}$ F. Oba, S. R. Nishitani, S. Isotani, H. Adachi, and I. Tanaka, J. Appl. Phys. 90, 824 (2001).

${ }^{10}$ D. V. Lang, J. Appl. Phys. 45, 3023 (1974).

${ }^{11}$ G. E. Pike and C. H. Seager, J. Appl. Phys. 50, 3414 (1979).

${ }^{12}$ M. A. Alim, M. A. Seitz, and R. W. Hirthe, J. Appl. Phys. 63, 2337 (1988).

${ }^{13}$ F. A. Modine, R. W. Major, S. I. Choi, L. B. Bergman, and M. N. Silver, J. Appl. Phys. 68, 339 (1990).

${ }^{14}$ D. Fernández-Hevia, J. de Frutos, A. C. Caballero, and J. F. Fernández, J. Appl. Phys. 92, 2890 (2002).

${ }^{15}$ I. M. Kaganova and M. I. Kaganov, Phys. Lett. A 173, 473 (1993).

${ }^{16}$ A. Rinkevich, A. Nossov, V. Ustinov, V. Vassiliev, and S. Petukhov, J. Appl. Phys. 91, 3693 (2002).

${ }^{17}$ K. S. Champlin, D. E. Armstrong, and P. D. Gunderson, Proc. IEEE 52, 677 (1964).

${ }^{18}$ O. V. Roos and K. L. Wang, IEEE Trans. Microwave Theory Tech. MTT34, 183 (1986).

${ }^{19}$ U. V. Bhapkar and T. W. Crowe, IEEE Trans. Microwave Theory Tech. MTT-40, 886 (1992).

${ }^{20}$ A. R. Hutson, Phys. Rev. 108, 222 (1957).

${ }^{21}$ K. I. Hagemark and L. C. Chacka, J. Solid State Chem. 15, 261 (1975).

${ }^{22}$ E. Ziegler, A. Heinrich, H. Oppermann, and G. Stöver, Phys. Status Solidi A 66, 635 (1981).

${ }^{23}$ G. D. Mahan, J. Appl. Phys. 54, 3825 (1983).

${ }^{24}$ F. A. Modine and R. B. Wheeler, J. Appl. Phys. 67, 6560 (1990).

${ }^{25}$ J. Fan and R. Freer, J. Appl. Phys. 77, 4795 (1995).

${ }^{26}$ C. W. Nan, A. Tschöpe, S. Holten, H. Kliem, and R. Birringer, J. Appl. Phys. 85, 7735 (1999). 